\title{
Tratamiento ortodóntico quirúrgico de canino maxilar impactado con reabsorción radicular bilateral: Reporte de caso.
}

Surgical orthodontic treatment of impacted maxillary canine with resorption bilateral radicular: Case report.

Sively Mercado-Mamani ${ }^{1, a}$, Katty Ríos-Villasis ${ }^{2, b}$.

\section{RESUMEN}

Los dientes caninos superiores permanentes impactados bilaterales son un problema clínico que puede provocar una serie de secuelas irreversibles como la reabsorción radicular de dientes vecinos. Se describe el caso de una paciente con ausencia clínica de caninos superiores que se encontraban impactados orientados hacia vestibular produciendo reabsorción de los dos tercios de la raíces de todos los incisivosSuperiores,los que fueron extraídos, en el mismo acto se realizó la exposición quirúrgica de los dos dientes caninos y posteriormente la tracción ortodontica de los caninos impactados, en el maxilar inferior se realizaron extracción de primeras premolares inferiores para compensar las extracciones que se realizaron en el maxilar superior.Ocho meses fueron necesarios para observar clínicamente las piezas impactadas. El tiempo de tratamiento fue de 2 años, al finalizar el tratamiento ortodoncicose realizó una ameloplastia de las cúspides de los caninos para dar forma de incisivos laterales.

Palabras clave: DIENTE IMPACTADO, REABSORCIÓN RADICULAR, MOVIMIENTO DENTARIO, DIENTE CANINO.

\begin{abstract}
The that were impacted oriented vestibular reabsorption producing 2/3 root of the upper incisors, they were extracted, with subsequent surgical exposure and orthodontic traction of the impacted canines in the lower jaw were made first premolars extraction, Camouflaging the alveolar biprotrusión. Eight months were required to observe clinically impacted teeth. The treatment time was 2 years old are ameloplastia cusps of the canines to build incisal edge.
\end{abstract}

Keywords: TOOTH, IMPACTED, ROOT RESORPTION, TOOTH MOVEMENT.

1 Universidad Católica Santa María. Arequipa, Perú.

2 Universidad Inca Garcilazo de la Vega. Lima, Perú.

a Magíster en Salud Pública. Especialista en Ortodoncia y Ortopedia Maxilar.

b Magíster en Estomatología. 


\section{INTRODUCCIÓN}

El diagnóstico precoz, la intercepción oportuna y un tratamiento multidisciplinario apropiado hacen posible que un canino maxilar impactado pueda ser posicionado dentro del arco dentario, de ahí,la importancia que los clínicos le han dado a este tema, debido a que es el diente con mayor frecuencia de impactaciónseguido por los terceros molares $(1,2)$.

La incidencia de caninos permanentes impactados superiores varía entre $0,92 \%$ a $2,2 \%$. $(3,4)$. Con relación al sexo son dos veces más común en mujeres $(1,17 \%)$ que en varones $(0,51 \%)(1)$.

La incidencia de impactaciónhacia el lado palatino es mayor con respecto al lado vestibular en una proporción de por lo menos 3:1 (5 ). El 8\% de los caninos impactados ocurren bilateralmente.

La etiología de los caninos impactados es multifactorial y difícil de definir. Hay que destacar que los caninos superiores están predispuestos a quedarse incluidos por presentar el periodo más largo de desarrollo, además de la vía de erupción más extensa y tortuosa. Mucho tiempo se especuló que los caninos maxilares tenían un trayecto más tortuoso que cualquier otro diente para su erupción y que esta situación estaba relacionada con tres factores: el tiempo requerido para su desarrollo, la posición cambiante durante su desarrollo y la distancia que recorre en su vía de erupción $(6,7)$.

Diversos factores locales y generales están relacionados con los caninos superiores impactados $(1,8,9,10)$. En el presente caso aparentemente hubo un factor local reconocido, que fue la retención prolongada de los caninos superiores .

Una de las causas que provocan la reabsorción radicular de dientes vecinos son los traumatismos, presión de los dientes permanentes en erupción, ello ha sido considerado como uno de los factores más importantes en la reabsorción radicular $(11,12)$.

El diagnóstico y tratamiento generalmente implica un abordaje multidisciplinario donde intervienen el cirujano maxilofacial, ortodoncista, periodoncista implantologo y Rehabilitador.

\section{REPORTE DE CASO}

Se reporta el caso de una paciente de sexo femenino de 15 años 9 meses de edad que acude al Servicio de Postgrado de ortodoncia de la Facultad de Odontología de la Universidad Católica de Santa María refiriendo que "sus dientes no le gustaban" porque los caninos superiores no habían erupcionado.

\section{Examen clínico.}

Paciente mesocéfala, braquifacial, con facie simétrica, con los tercios faciales proporcionados, perfil ligeramente convexo hipodivergente (Fig. 1A). Al evaluar la oclusión presenta una relación molar clase I bilateral, relación canina bilateral no registrables, over jet $5 \mathrm{~mm}$, over bite $5 \%$. Los arcos dentales son ovalados, el superior espaciado moderado (6mn)el arco inferior apiñado moderado (5 $\mathrm{mm})$. La línea media superior se encuentra desviada $1.5 \mathrm{~mm}$. hacia el lado derecho, la línea media inferior está desviada $3 \mathrm{~mm}$ a la izquierda, ausencia clínica de piezas 13 y 23 y movilidad dentaria de grado 2 en piezas 11,12, 21, 22 (Fig. 1B).

\section{Exámenes auxiliares:}

En las radiografíasperiapicales anterosuperiores muestran las piezas 13 y 23 impactadas que producían reabsorciónde aproximadamente $2 / 3$ radiculares de los incisivos centrales superiores y casi $3 / 3$ de los incisivos laterales (Fig. 2A). Mediante la técnica de desplazamiento horizontal de Clark, ambas piezas se encuentran con la porción coronal orientada hacia vestibular y la radicular hacia palatino.

En la radiografía panorámica se observan estructuras óseas normales, piezas dentarias permanentes, impactadas 13 y 23 con formación completa de raíz e inclinación coronaria mesial, reabsorción radicular severa de todos los incisivos superiores, así como inclusión dela pieza 3.5 (Fig. 2B). Cefalométricamente existía una relación esquelética Clase I con ligera protrusión maxilar y biprotrusión dentaria superior e inferior (Fig. 2C). El análisis de modelos hay una discrepancia alveolo dentaria (DAD) superior de $-18.5 \mathrm{~mm}$, en el maxilar inferior $-7.5 \mathrm{~mm}$, Bolton total de $-5.2 \mathrm{~mm}$ en el maxilar superior.

\section{Diagnóstico:}

Mal oclusión Clase I, con caninos superiores 
impactados bilateralmente localizados a nivel apical de todos los insicivos superiores, con reabsorción radicularsevera, relación molar bilateral clase I y línea media superior desviada $1.5 \mathrm{~mm}$ a la derecha, la línea media inferior desviada $3 \mathrm{~mm}$ a la izquierda.

\section{Plan de tratamiento:}

Se realizan extracciones de los incisivos superiores, exposición quirúrgica y colocación de botones para tracción ortodónticade caninos impactados, extracciones de los primeros premolares inferiores. Tratamiento ortodóncico utilizando Técnica de Roth, aparatología fija $0.022 \times 0.028$ y uso de anclaje reciproco.

\section{Tratamiento.}

En una sola sesión se realizo las exodoncias de los incisivos laterales superiores, se realizo la exposición quirúrgica de los caninos superiores, en la cual se colocaron botones adhesivos para la tracción de los caninos expuestos quirúrgicamente, se preservaron los incisivos centrales con fines estéticos mismos que fueron ferulizados por palatino con resina. (Fig. 3a, $b, c)$.

En el maxilar inferior se extrajeron las primeras premolares inferiores. En la arcada superior los caninos se fueron traccionando inicialmente con ligadura metálica. Después de 8 meses de tracción se cementaron brackets a los caninos, colocando en la arcada superior un arco de activación térmica y se realizó control radiográfico. (Fig. 4a).Culminada la tracción canina, estos fueron contorneados a la forma de incisivos laterales superiores, los incisivos centrales fueron extraídos (Fig. 4b) colocando dientes provisionales ferulizados con fines estéticos. (Fig.4c). El alineamiento y nivelación del arco dental superior e inferior se realizó siguiendo una mecánica de fuerzas ligeras. Después de 2 años de iniciado el tratamiento se retiraron los brackets. Luego se recontornearon las cúspides de los caninos y mediante el uso de resina se modificó la forma de estos dientes para semejarlos a incisivos laterales.. Adicionalmente se evaluó la condición gingival de las piezas traccionadas. Para la fase de contención se utilizaron placas removibles continuas, realizando la derivación de la paciente al área de Implantologia (Fig 5).

\section{DISCUSIÓN}

La erupción vestibular del canino maxilar está fuertemente asociada con la falta de espacio o el apiñamiento del arco alveolar, largos periodos de erupción, discrepancias del arco dentario maxilar, posiciones anormales, anquilosis, trauma o alteraciones patológicas como quistes entre otras, así como el desplazamiento del folículo dentario, aumento de la presión intravascular dentro del germen dentario, falla en la reabsorción radicular del canino temporal e incluso procesos infecciosos crónicos (13).

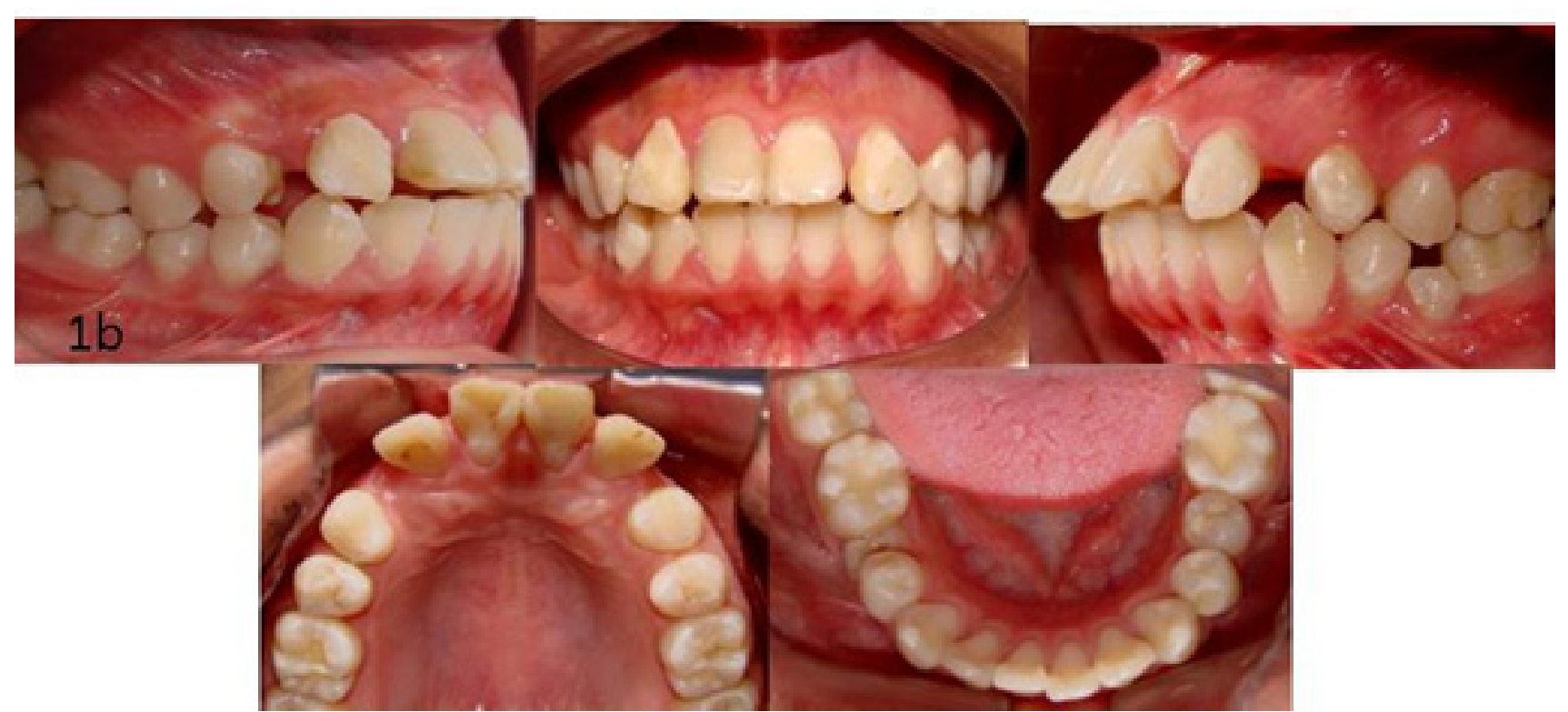

Fig 1. Fotografías Iniciales 1A. Fotografía lateral derecha, R.M: Clase I, R.C: no registrable, ausencia pieza 13, línea media alterada, OB: alterado, ausencia de piezas 13, 23. Fotografía lateral Izquierda, RM: Clase I, RC: no registrable, OJ: alterado. 


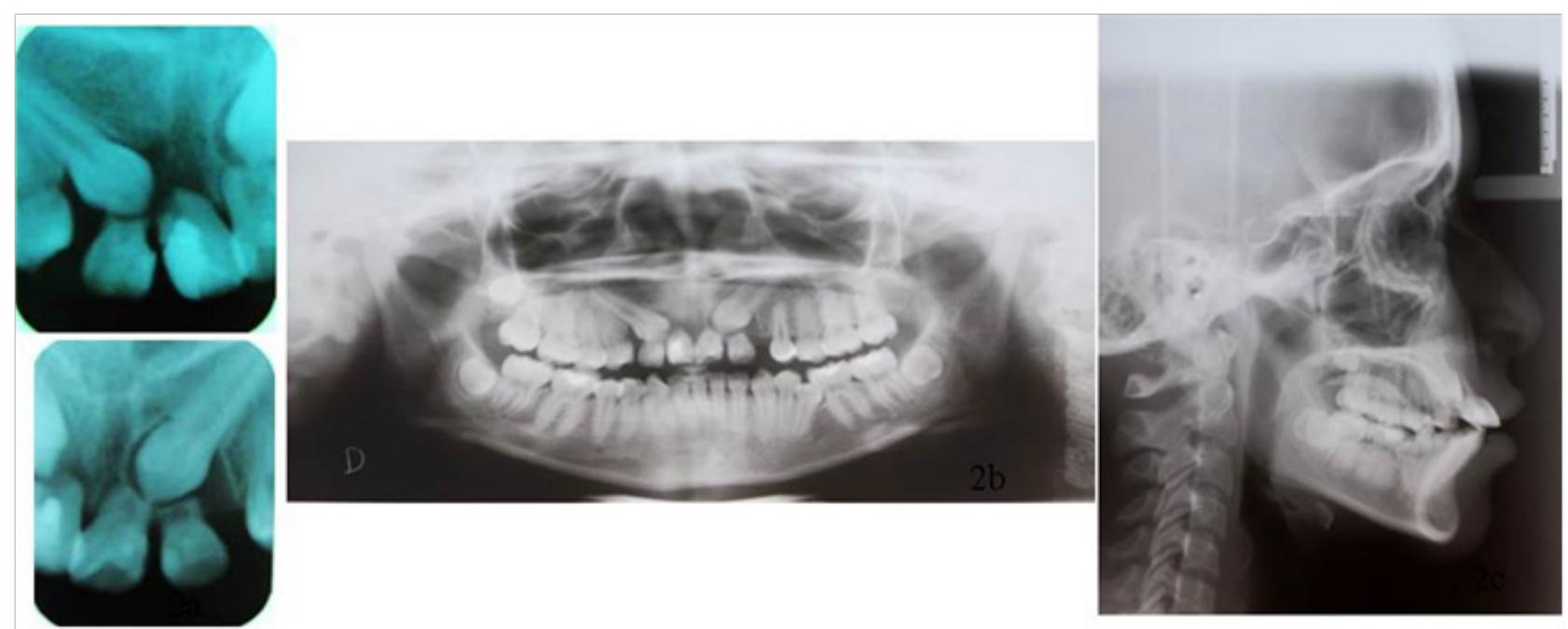

Fig. 2A. Radiografía periapical anterosuperior. 2B. Radiografíapanorámica. 2C. Radiografía cefalométrica.

El 2\% de la población puede tener caninos superiores impactados, y esta condición es dos veces más frecuente en mujeres que en hombres (14-15).

La incidencia de impactación canina es dos veces más que en la mandíbula,donde aproximadamente un tercio de los caninos maxilares impactadosse encuentran en posición vestibular, y dos tercios se encuentran en posición palatina (16-17).

El enfoque más conveniente para el manejo de los caninos superiores impactados es el diagnóstico precoz y su intercepción, debido a la escaza prevención de este cuadro es necesario considerar el tratamiento de ortodoncia seguido de la exposición quirúrgica de los caninos para ser llevados a una oclusión normal, por tanto la relación del diente con las estructuras anatómicas es un factor importante a considerar. (18), el tratamiento quirúrgico ortodontico.fue de elección ya que no existe el riesgo de comprometer elementos neurovasculares importantes. Es importante tener en cuenta que esta no se pueda llevar a cabo si existe una patología asociada a los dientes impactados (quistes voluminosos, infección, rizólisis, necrosis pulpar) o una enfermedad sistémica grave .El hallazgo de un canino superior incluido e impactado en un paciente adulto representa un manejo terapéutico especial, ya que al ser diagnosticado a edades más avanzadas, pueden provocar problemas irreversibles :como la reabsorción radicular severa de piezas vecinas.(19)

En nuestro caso, debido a las condiciones locales se decide realizar la cirugía para tracción ortodontica. Según Ericson\&Kurol la impactación vestibular tiene una angulación vertical más favorable, a diferencia de la impactación palatina que es más horizontal. Se optó por realizar la técnica cerrada mediante un colgajo vestibular de reposición completa junto a la técnica de erupción abierta, la cual consiste en la realización de un colgajo vestibular mucogingival de espesor completo en forma de $U$ teniendo en consideración la preservación de papilas para los dientes vecinos (14).

Además, la técnica de colgajo vestibular de reposición completa tiene la ventaja de generar la

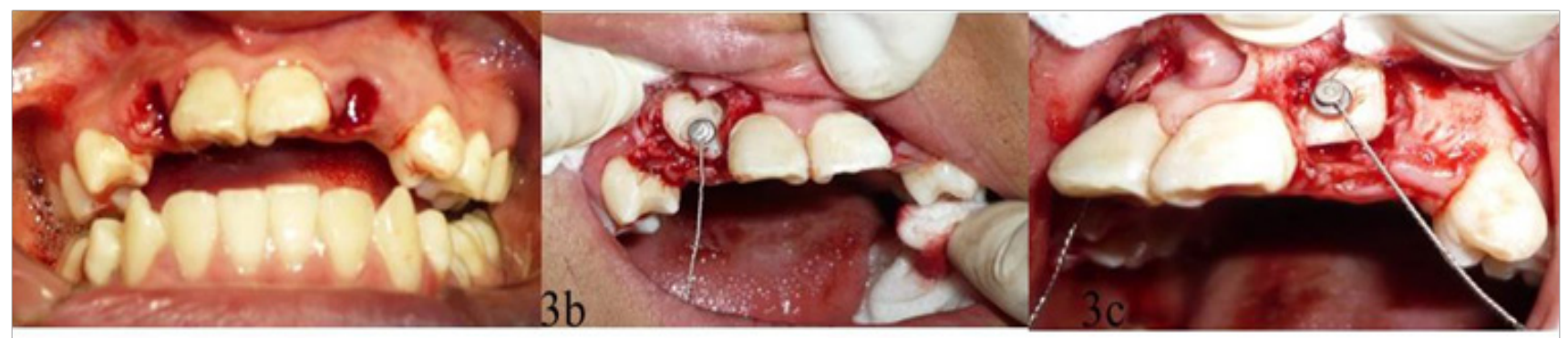

Fig. 3. Exposición quirúrgica de los caninos superiores 

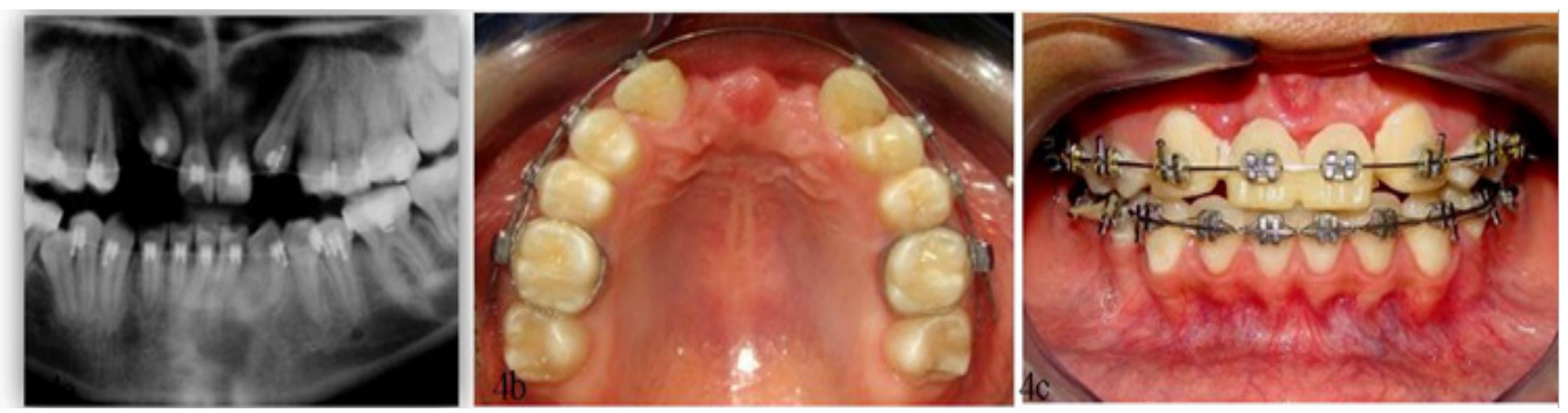

Fig 4A. Radiografía de control, tracción canina bilateral, 4B. Caninos en plano oclusal, extracción de ICS, 4C. Dientes provisionales ferulizados.

mejor estética gingival y una mayor facilidad de movimiento de los dientes (14-20).

Los caninos superiores bilateralmente impactados fueron alineados con éxito sin afectar adversamente el periodonto. Se observó una notable mejora en la estética facial y la oclusión dental. (Fig.5) Aunque los dientes impactados habían provocado una reabsorción severa de los incisivos superiores (Fig.2a, $2 b)$. Se mantuvo un buen control vertical durante el tratamiento.

La compensación dental planeada fue exitosa para camuflar la biprotrusión dentaria que presentaba, la paciente al finalizar el tratamiento tenía un buen equilibrio facial, y sonrisa consonante. El estado periodontal de los caninos superiores fue estable.

Se ha enfatizado en la complejidad de manejar dientes desplazados hacia vestibular sin que ocurran efectos adversos periodontales, por lo que es fundamental una cuidadosa técnica quirúrgica, una colocación de encía marginal, el control de la inflamación, la ausencia de una fuerza excesiva y la adherencia gingival (21).

La evidencia actual no ha logrado diferenciar que la técnica abierta es diferente de un injerto posicionado apicalmente, es decir, erupcion abierta, frente a la erupción cerrada, aunque existen informes contradictorios sobre el tejido queratinizado posicionado apicalmente y la técnica erupción cerrada utilizada para descubrir los dientes en posición ectópica (22).

El manejo de los caninos maxilares impactadoses importante en términos de estética y función. Este procedimiento permite por lo general a los caninos una erupción correctamente en el arco dental. En cuanto al tipo de cirugía, el pronóstico estará condicionado tanto por la posición vertical del canino impactado como por la calidad del tejido queratinizado.

\section{CORRESPONDENCIA}

Sively Mercado Mamani

Dirección: Urb. Huáscar C-16; Puno.

Teléfono: 511-989071293

Email: goty20@hotmail.com

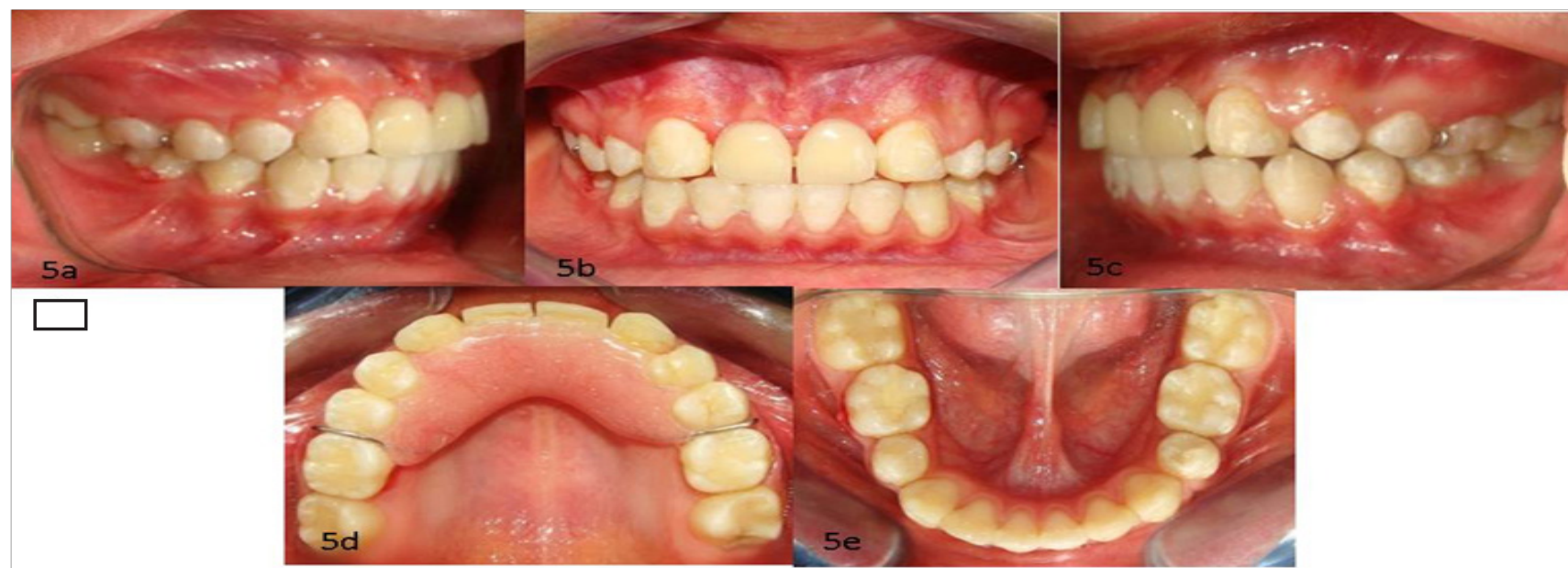

Fig. 5. Fotografías finales 


\section{REFERENCIAS BIBLIOGRÁFICAS}

1. Bishara SE. Impacted maxillary canines: a review. Am. J. Orthod. Dentofacial.Orthop. 1992;101(2):159-71.

2. Ngan P,Hornbrook, R, Weaver B.Early timely management of ectopically erupting maxillary canines. Semin.Orthod. 2005;11(3):152-63.

3. Bishara SE, Comer DD, McNeil MH, Montagano LN, Oesterle LJ, Yoongquist HW. Management of impacted canines. Am J. Orthod. 1976;69 (4):371-87.

4. Ericson S, Kurol J. Radiographic assessment of maxillary canine eruption in children with clinical signs of eruption disturbances. Eur J Orthod. 1988;8(3):133-40.

5. Fournier A, Turcotte JY, Bernard C. Orthodontic considerations in the treatment of maxillary impacted canines. Am J Orthod 1982;81(3):236-9.

6. Dewel BF.The upper cuspid: Its development and impaction. Angle Orthod 1949;19:79-90.

7. Jacoby $\mathrm{H}$. The etiology of maxillary canine impactions. Am J Orthod 1983;84(2):125-32.

8. Becker A, Bimstein E, Shteyer A. Interdisciplinary treatment of multiple unerupted supernumerary teeth. Report of a case. Am J Orthod 1982;81(5): 417-22.

9. Lewis PD. Preorthodontic surgery in the treatment of impacted canines. Am J Orthod. 1971;60(4):382-97.

10.Linge L. Patients charasteristics and treatment variables associated with apical root resorption during orthodontic treatment. Am J Orthod. 1992;35-43.

11.-Hitoshi S. Root resorption of upper permanent incisor, caused by impacted canine. Am J Orthod. 1984;299-306.
12.Riyad A. Genetic predispositition to external apical root resorption: linkage ofchomosome-18 market. J Dent Res. 2003;82(5):356-60.

13.Mochizuki, K. The horizontally impacted situated in a labial position. Bull.Tokio Dent. Coll 1999;40(4):203-8.

14. Cooke J. Canine impactions: incidence and management. Int. J. Periodontics Restorative Dent. 2006;26:483-91.

15.Proffit, WR, Fields H, Sarver D. ContemporaryOrthodontics.4th ed. Mosby, St. Louis, 2007.

16. Yavuz, M. S. Impacted mandibular canines. J. Contemp. Dent. Pract. 2007;8(7):78-85.

17.Bedoya MA. Review of the diagnosis and management of impacted maxillary canines. J. Am. Dent.Assoc 2009; 140:1485-93.

18.Flores R. Inserción de un implante simultaneo a la extracción de un canino superior incluido. Avances en Periodoncia. 2007;19(2):85-9.

19.Magheri P. Restorative alternatives for the treatment of an impacted canine: surgical andprosthetic considerations. Pract.Proced.Aesthet. Dent. 2002;14(8):659-64.

20.Kokich V. Surgical and orthodontic management of impacted maxillary canines. Am. J. Orthod. DentofacialOrthop 2004;126(3):278-83.

21.Vanarsdall RL. Efficient management of unerupted teeth: A time-tested treatment modality. Semin.Orthod. 2010;16(3):212-21.

22.Schubert M. Alignment of impacted maxillary canines: critical analysis of eruption path and treatment time. J. Orofac. Orthop. 2009;70(3):200-12.

Recibido : 07-03-2012

Aceptado: 11-07-2012 